\title{
High-Resolution, High-Contrast Optical Interface for Defect Qubits
}

\author{
Jong Sung Moon, ${ }^{\ddagger}$ Haneul Lee, ${ }^{\ddagger}$ Jin Hee Lee, Woong Bae Jeon, Dowon Lee, Junghyun Lee, \\ Seoyoung Paik, Sang-Wook Han, Rolf Reuter, Andrej Denisenko, Jörg Wrachtrup, Sang-Yun Lee, \\ and Je-Hyung Kim*
}

Cite This: ACS Photonics 2021, 8, 2642-2649

Read Online

ACCESS | W Wetrics \& More | 回 Article Recommendations

ABSTRACT: Point defects in crystals provide important building blocks for quantum applications. Since we optically address these defect qubits, having an efficient optical interface is a highly important aspect. However, conventional confocal fluorescence microscopy of high-refractive-index crystals suffers from limited photon collection efficiency and spatial resolution. Here, we demonstrate high-resolution, high-contrast imaging of defects in diamonds using microsphere-assisted confocal microscopy. A microsphere provides an excellent optical interface for point defects with a magnified virtual image that increases the spatial resolution up to $\lambda / 5$, as well as the optical signal-to-noise ratio by four times. These features enable individual optical addressing of single photons and single spins of multiple defects that are spatially unresolved in conventional confocal microscopy, with improved signal contrast. Combined with

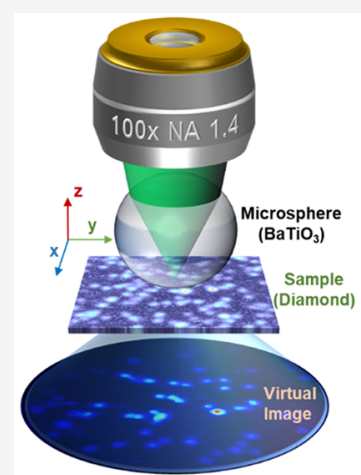
Supporting Information optical tweezers, this system also demonstrates the possibility of positioning or scanning the microspheres. The approach does not require any complicated fabrication or additional optical systems, but uses simple, off-the-shelf micro-optics. From these distinctive advantages of microspheres, our approach provides an efficient way to image and address closely spaced defects with much better resolution and sensitivity.

KEYWORDS: point defects, microsphere-assisted microscopy, single photons, optically detected magnetic resonance, quantum sensing

$\mathrm{O}$ ptically active point defects in crystals such as nitrogenor silicon-vacancy centers in diamond have emerged as the most attractive candidates for implementing quantum technologies in solid-state platforms. ${ }^{1,2}$ These defects can provide single photons and a long coherent spin over $1 \mathrm{~ms}$ at room temperature. ${ }^{3,4}$ Recently, beyond single defects, addressing multiple adjacent defects has become increasingly important. A number of groups have demonstrated scalable quantum systems that enable multiqubit registers, ${ }^{5,6}$ superradiant emissions from multiple resonant emitters, ${ }^{7,8}$ and widefield imaging of magnetic or electric fields. ${ }^{9-13}$ Therefore, to optically address multiple adjacent defects, an optical interface with high resolution and high readout contrast is of paramount importance. However, accessing the defects in bulk crystals using conventional confocal microscopy, which consists of a high numerical aperture (NA) objective lens with a pinhole for spatial filtering, lacks such capabilities due to diffraction-limited spatial resolution and background fluorescence from crystals, substrates, and nearby defects.

There have been many efforts to improve the light extraction efficiency by fabricating micro/nanophotonic structures such as nanowires, ${ }^{14}$ nanobeams, ${ }^{15}$ bullseyes, ${ }^{16}$ and photon extractors with inversed designs. ${ }^{17}$ These structures have dramatically increased the brightness of single defects by more than an order of magnitude and enhanced the radiative recombination rate by the Purcell effect. ${ }^{18}$ However, these approaches require a sophisticated fabrication process for materials with high hardness, such as diamond, and nanoscale devices often aggravate the coherence properties of photonic and spin qubits. ${ }^{19-21}$ Furthermore, to deterministically couple defects with photonic structures in the maximum optical mode, precise spatial control of photonic structures or defects is required. $^{22-25}$

To improve the spatial resolution, various super-resolution techniques, such as near-field probes, ${ }^{26}$ plasmon gratings, ${ }^{27}$ stimulated emission depletion microscopy, ${ }^{28,29}$ and Fourier magnetic imaging, can be employed. ${ }^{30}$ These techniques have led to a significant improvement in the spatial resolution of less than $10 \mathrm{~nm}$, but they are still not widely used for studying defects due to several issues: limited imaging depth, low optical throughput, nonradiative loss, and complicated imaging systems, combined with a depletion beam or a gradient

Received: April 17, 2021

Published: August 19, 2021 


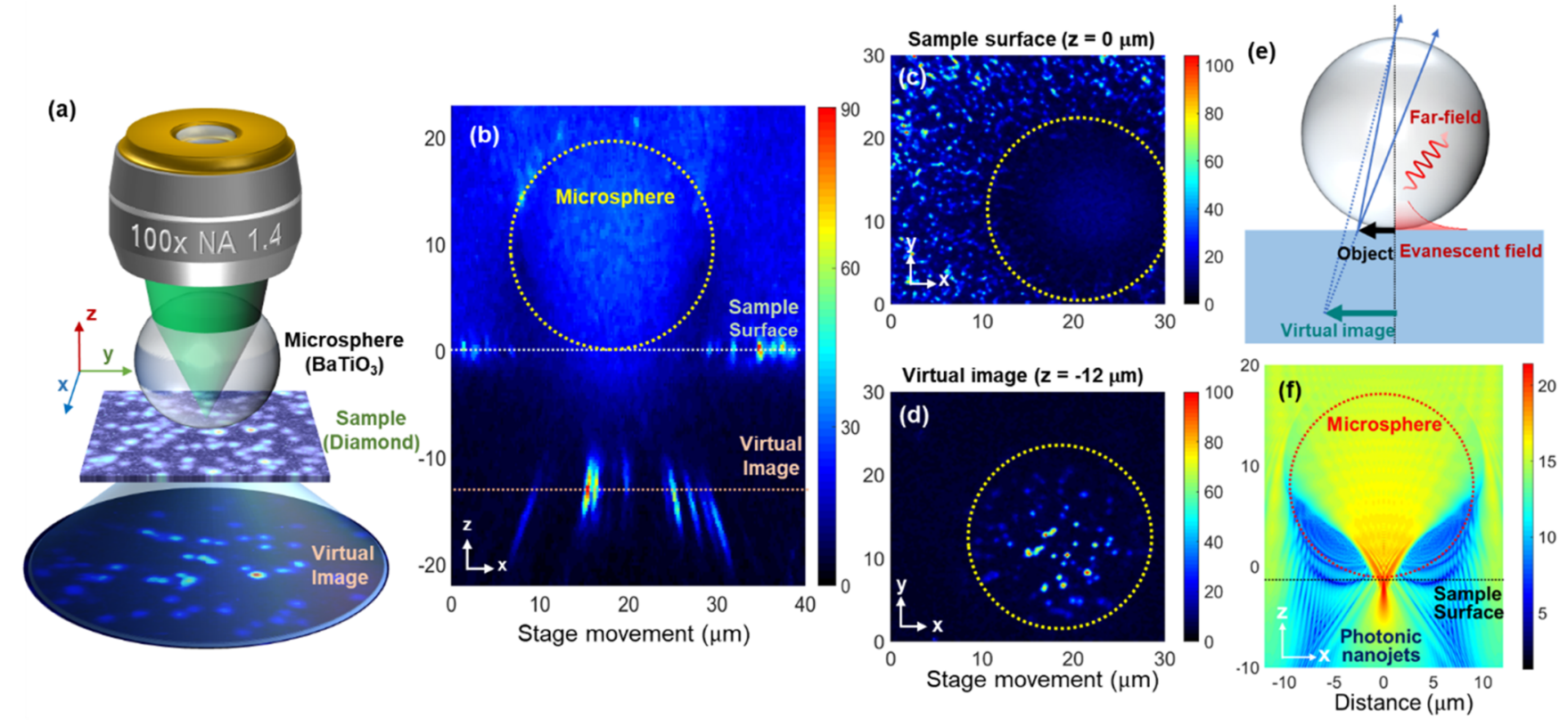

Figure 1. (a) Schematic description of microsphere-assisted microscopy with a virtual image underneath the sample surface. (b) Vertically scanned confocal image of defects with a microsphere. The dotted lines indicate the microsphere, sample surface, and virtual image plane. (c, d) $x-y$ scanned confocal images with the microsphere at different vertical planes of $z=0 \mu \mathrm{m}$ (sample surface) (c) and $z=-12 \mu \mathrm{m}$ (virtual image plane) (d). The color bar denotes the photoluminescence (PL) intensity in units of k counts per second. (e) Schematic representation of the optical phenomenon in the microsphere that magnifies the image and couples the evanescent field. (f) Numerical simulation of an incident plane wave toward a $20 \mu \mathrm{m}$ sized microsphere on diamond. A logarithmic color map of the intensity $\left(|E|^{2}\right)$ shows the photonic nanojet effect that tightly focuses the incident wave at the bottom of the microsphere.

magnetic field. Therefore, addressing closely spaced defects with high spatial resolution and high readout contrast remains a challenge.

Here, we introduce a high-resolution, high-contrast optical interface for defects based on hybrid integration of dielectric micro-optics. Dielectric microspheres have several advantages over previously reported fabrication-based photonic structures and super-resolution techniques. First, the microspheres are off-the-shelf optical elements with a wide range of options regarding their materials and sizes. We can couple these optics to defects by simple dispersion. The hemisphere solid immersion lenses could also be considered as micro-optics, and they have demonstrated large enhancement in the brightness, ${ }^{31-34}$ improved spatial resolution, ${ }^{35}$ and capability of position control. ${ }^{35,36}$ However, the microsphere has distinctive advantages over solid immersion lenses. The microspheres enable super-resolution imaging beyond the conventional diffraction limit of a high NA objective lens by subdiffraction-limited illumination and far-field propagation of evanescent waves. ${ }^{37-40}$ In particular, the microspheres form virtual or real images far apart from the actual sample surface. ${ }^{41}$ This spatially translated image separates the defect signals from optical background noise existing near the sample surface. Such capabilities of high-resolution, high-contrast optical imaging enable us to optically address single photons and single spins of defects that are spatially unresolved in conventional confocal microscopy. Furthermore, the microsphere can be optically controlled using optical tweezers for deterministic positioning and wide-field scanning of defects. Therefore, together with a simple, fabrication-free setup, the microspheres provide a relocatable and efficient optical interface for solid-state quantum emitters.

\section{RESULTS AND DISCUSSION}

We prepared point defects in diamond by ion implantation of $\mathrm{N}_{2}$ ions that created randomly distributed nitrogen-vacancy $(\mathrm{NV})$ color centers near the surface within $100 \mathrm{~nm}$ with a density of $1 \sim 2 \mu \mathrm{m}^{-2}$. For microsphere-assisted confocal fluorescence microscopy, we dispersed $\mathrm{BaTiO}_{3}$ microspheres on the diamond sample. The $\mathrm{BaTiO}_{3}$ microspheres have a high refractive index $(n \sim 1.9-2.1)$ and a diameter of $10 \sim 20 \mu \mathrm{m}$. The high-refractive-index sphere is more beneficial for imaging defects in diamond $(n \sim 2.42)$ than other low-index microspheres such as soda-lime glass $(n \sim 1.51)$ and polystyrene $(n \sim 1.59)$ since the small refractive index contrast between the microsphere and diamond can minimize the total internal reflection at the interface. The refractive index contrast between the microsphere and its surrounding environment plays an important role in the image, as it determines the propagation of light through the microspheres. A small (large) index contrast with the environment forms a virtual (real) image below (above) the sample. ${ }^{41}$ To maintain the advantage of high-index microspheres but reduce the index contrast with the environment, we immersed the microspheres in immersion oil $(n=1.518)$. By doing so, we can bring the capability of super-resolution imaging to a virtual image plane. ${ }^{39-42}$ Furthermore, the small index contrast between the spheres and immersion oil reduces the scattering force at the interfaces and allows us to trap the high-index microsphere using optical tweezers. For fluorescence imaging, we used a home-built confocal microscopy setup at room temperature. We excited the sample with a $532 \mathrm{~nm}$ continuous-wave laser and collected the fluorescence from the sample with an oil immersion objective lens $(\mathrm{NA}=1.4)$. We spectrally filtered out the exciting laser from the collected fluorescent signals by using a dichroic mirror and then spatially filtered out the emission by 
using a $50 \mu \mathrm{m}$ pinhole and two lenses, followed by a fibercoupled silicon single-photon avalanche photodiode (SPCMAQRH, Excelitas).

Figure 1a illustrates the microsphere-assisted microscopy for imaging defects in diamond. Immersing the high-refractiveindex microsphere in a liquid $(n \sim 1.518)$ forms a virtual image of defects underneath the sample surface. We first vertically scan the sample to determine image planes with and without a microsphere lens. As shown in Figure $1 \mathrm{~b}$, outside the microsphere, the confocal map displays the fluorescence from defects at $z=0$ near the diamond surface, whereas the defects under the microsphere create a virtual image at a lower plane near $z=-12 \mu \mathrm{m}$. Figure $1 \mathrm{c}, \mathrm{d}$ exhibits $x-y$ lateral maps at two different image planes of $z=0$ and $-12 \mu \mathrm{m}$. In comparison, the virtual image in Figure 1d noticeably shows defects with better contrast and lateral resolution than those at the sample surface outside the microsphere. While the photonic nanojet tightly illuminates the defects in the lateral direction, its vertically elongated shape results in extended defect images along the $z$-direction at the virtual plane.

The improvement in the image quality is relevant to the optical phenomena in the microsphere. As described in Figure 1e, a microsphere, as a spherical lens, creates a magnified virtual image. The magnification factor varies with the size of spheres, the optical index contrast, and the distance from the microsphere to the defects. ${ }^{37,40,43}$ More importantly, the microsphere improves the spatial resolution beyond the diffraction limit in conventional confocal microscopy. ${ }^{37-40,44}$ First, the microsphere couples high-frequency evanescent waves to far-field emission within the gap $(\lambda / 2)$ between the microspheres and the surface, as described in Figure 1e. This coupled near-field enhances both the light extraction and spatial resolution, which occur in the vicinity of the contact area around one-quarter of the sphere size. ${ }^{45}$ Another important phenomenon is subdiffraction-limited illumination, the so-called photonic nanojet. ${ }^{40,44,46,47}$ Figure if shows a finite-difference time-domain simulation of cross-sectional $|E|^{2}$ profiles with a $20 \mu \mathrm{m}$ diameter microsphere. The dielectric microsphere leads to constructive interference between diffracted waves. ${ }^{40}$ In the simulation, the microsphere tightly focused the incident wave into a localized spot with a beam waist of $232 \mathrm{~nm}$ for an illuminating wavelength of $532 \mathrm{~nm}$. Previously, from these effects on collection and excitation, microsphere-assisted microscopy demonstrated the superresolution capability for $50 \mathrm{~nm}$ sized nanoparticles ${ }^{39}$ and 75 nm sized virus samples. ${ }^{37}$

For quantitative analysis, we directly compare the images of the same defects with and without the microsphere. This comparison was possible since the microspheres were relocatable. ${ }^{48-51}$ To change the position of microspheres, we combined optical tweezers (see Figure S1 and Supporting Video 1 in the Supporting Information).

Figure 2a,b displays the lateral confocal maps with/without the microsphere. In the same stage scan range, the microsphere shows a magnified virtual image that is almost doubled in size. We determine the magnification factor $(M)$ of the virtual image from the nonmagnified confocal image without the microsphere. Figure $2 \mathrm{c}$ shows the experimentally measured $M$ with depth. The magnification increases from 1.9 to 2.5 in the range of $z=-9 \sim-15 \mu \mathrm{m}$. The magnification in the spherical lens system can be approximated using geometrical optics. ${ }^{37}$ By considering a spherical aberration, the microsphere has a focal length $f(x)=\frac{x}{\sin \left[2 \sin ^{-1}(x / R)-2 \sin ^{-1}\left(n_{\mathrm{r}}^{-1}(x / R)\right)\right]}$, where $x$ is
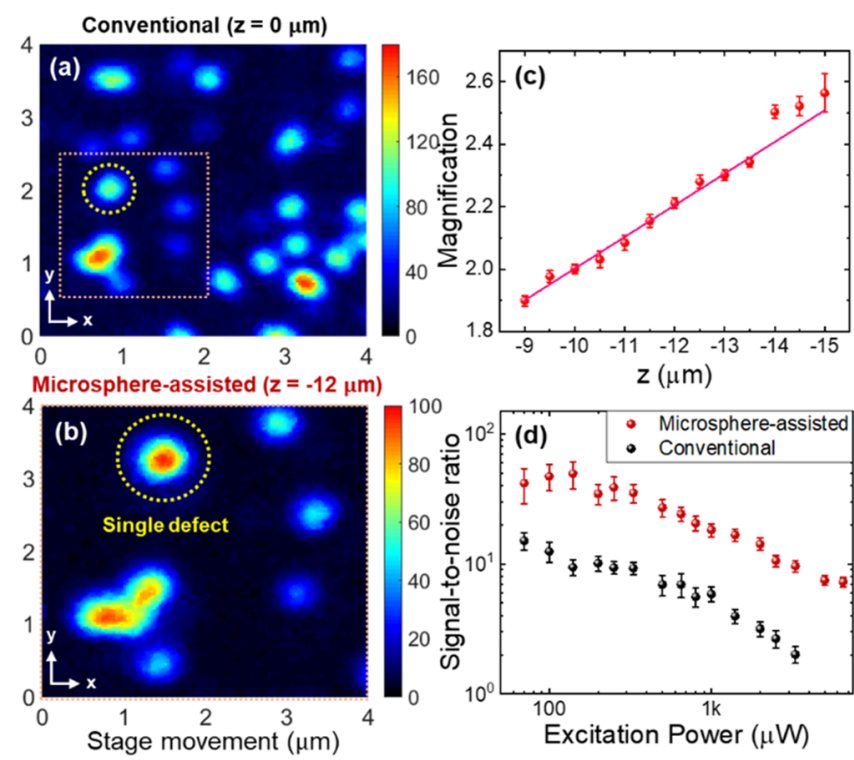

Figure 2. (a, b) Comparison of confocal fluorescence images of (a) conventional and (b) microsphere-assisted microscopy. Two images are laterally scanned in the same $x-y$ scan range at $z=0$ (without a microsphere) and $z=-12 \mu \mathrm{m}$ (with a microsphere). The magnified image in (b) corresponds to the area, marked as the orange-dashed box in (a). (c) Magnification factors measured at different virtual image planes in microsphere-assisted microscopy. A solid line denotes a calculated magnification factor $M(z)$. (d) Comparison of the signalto-noise ratio with (red) and without (black) the microsphere. The yellow circles in (a) and (b) denote the single defect used for the comparison in $(\mathrm{d})$.

the transverse distance from the optical axis, $R$ is the radius of a microsphere, and $n_{\mathrm{r}}=\frac{n_{\text {microsphere }}}{n_{\text {oil }}}=1.38$ is the refractive index contrast between the microsphere and the oil. Then, the sphere creates a virtual image at a distance $\left(d_{\mathrm{v}}=\frac{(R+\delta) f}{f-R-\delta}-R\right)$ from the sample surface. The magnification is given as $M=\frac{R+d_{\mathrm{v}}}{R+\delta}$, where $\delta$ is the depth of defects from the sample surface. Following the above formula with $R=10 \mu \mathrm{m}, \delta=100 \mathrm{~nm}$, and $x=1 \mu \mathrm{m}$, we can calculate the virtual image plane at $d_{\mathrm{v}}=-13$ $\mu \mathrm{m}$, with $M=2.28$. Depending on the observed virtual plane, the magnification varies as $M(z)=\frac{R-z}{R+\delta}$. The theoretically calculated values are well matched to the experimentally observed results shown in Figure 2c.

In terms of brightness, the high-index spheres couple the evanescent waves near the diamond surface to free space. From the numerical simulation, we calculate the improved extraction efficiency of dipoles under the microspheres to be a factor of 2 . In the experiment, we observe an enhancement in brightness up to $40 \%$ by comparing the saturation intensity of the same defect with and without a microsphere (see Figure S2 in the Supporting Information). The discrepancy between the simulation and observation is likely due to the narrow spatial window of the microsphere for the maximum brightness. The simulation shows that the maximum enhancement only occurs within approximately $1 \mu \mathrm{m}$ under the center of the sphere (see Figure S3 in the Supporting Information). This window can be widened by increasing the size of the microsphere.

Although the brightness enhancement is moderate, the microsphere enables a large increase in the signal-to-noise ratio with substantially reduced background fluorescence at the 

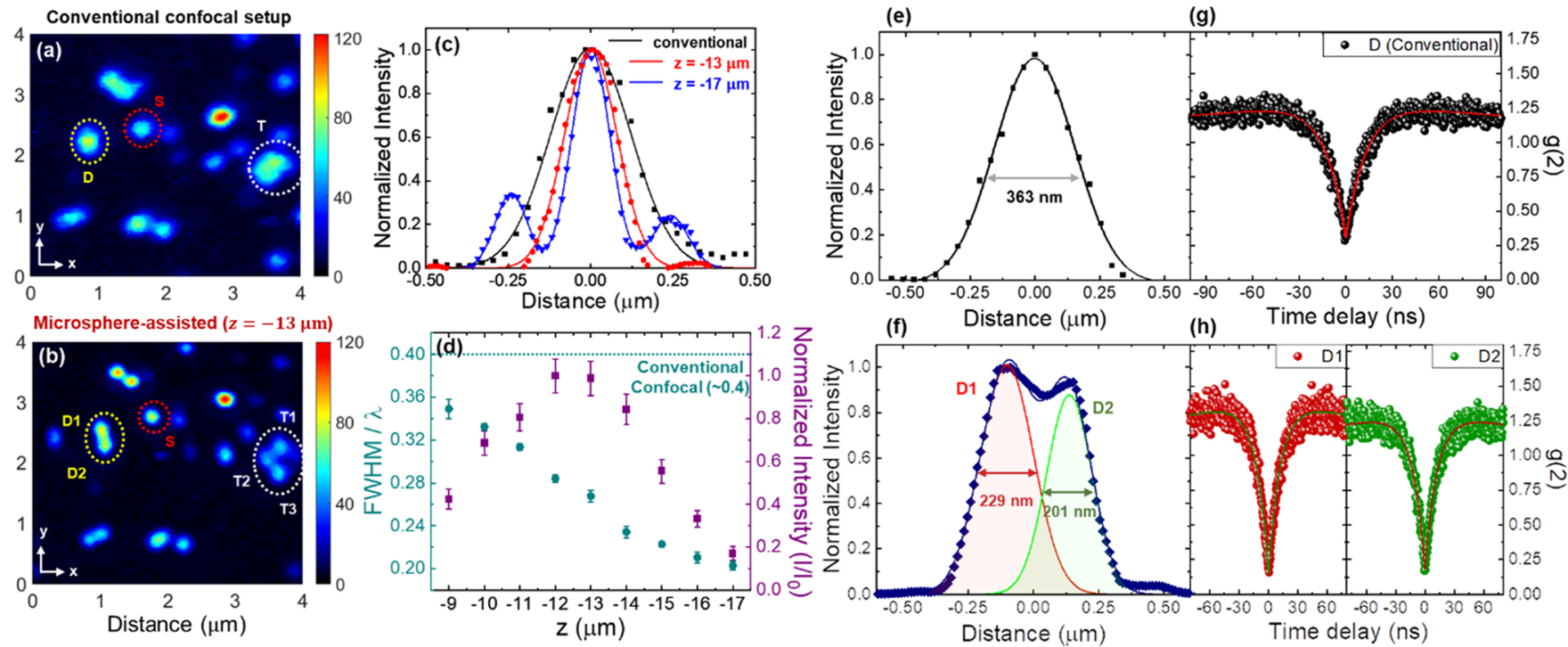

Figure 3. (a, b) Confocal images of defects in (a) conventional and (b) microsphere-assisted microscopy. The virtual image at $z=-13 \mu \mathrm{m}$ in (b) is rescaled with the magnification factor to compare with the conventional confocal image in (a). Defects labeled S, D, and T in (a) denote single defects and spatially unresolved double and triple defects, respectively. These defects are well separated in (b) and labeled D1 and D2 and T1-T3. (c) Cross-sectional intensity profiles of defect $\mathrm{S}$ from conventional confocal microscopy (black dots) and microsphere-assisted microscopy at different $z$ positions of $z=-13$ (red dots) and $-17 \mu \mathrm{m}$ (blue dots). Solid lines are fitted lines with a Gaussian function. (d) Spatial resolution and normalized intensity of the single defect $S$ as a function of the $z$ position of the virtual image. A green-dashed horizontal line indicates the diffraction limit of conventional microscopy. (e, f) Intensity profiles of two adjacent defects D in (a) and D1 and D2 in (b). The data in (e) and (f) are fitted with single- and two-separated Gaussian functions, respectively. $(\mathrm{g}, \mathrm{h}$ ) Second-order photon correlation curves, measured at ( $\mathrm{g}$ ) the maximum peak of the merged defect D and (h) each peak of the separated defects D1 and D2. The red lines are fitted $g^{(2)}(\tau)$ curves.

virtual image plane compared to that at the sample surface. The optical background comes from the fluorescence of the sample, such as the immersion oil, host crystal, and nearby defects. Besides, the use of oil increases the background signals due to largely enhanced scattering. The background signal is much brighter above near the diamond surface compared to the region below the diamond, as observed in Figure $1 \mathrm{~b}$. Therefore, spatial separation of the virtual image far below the sample surface is beneficial to reduce the optical background noise. Achieving a high signal-to-noise ratio is very important for solid-state qubit systems since we need to manipulate the signals of single photons. Figure $2 \mathrm{~d}$ compares the signal-tonoise ratio of the same single defect (yellow circles in Figure $2 \mathrm{a}, \mathrm{b})$ with and without the microsphere. The results shows a large enhancement in the signal-to-noise ratio by an average of four times for the virtual image.

The fluorescence of a single atomic defect can serve as a perfect point-spread function for measuring the spatial resolution of the image. To determine the spatial resolution of this microsphere-assisted microscopy approach, we calibrate the scale of the virtual image using the measured $M$. In Figure $3 \mathrm{a}, \mathrm{b}$, the spatially unresolved defect groups $\mathrm{D}$ and $\mathrm{T}$ in conventional microscopy are well resolved as separated defects by microsphere-assisted microscopy, with labels D1 and D2 and T1-T3, respectively. The result clearly shows the improved resolving power of microsphere-assisted microscopy. To compare the full width at half-maximum (fwhm) of the point-spread function, we select the single defects labeled $S$ in Figure $3 a, b$ and plot the cross-sectional intensity curve in Figure $3 \mathrm{c}$. In conventional microscopy, the fwhm of the fitted Gaussian function is approximately $280 \mathrm{~nm}$. Considering the center wavelength of $700 \mathrm{~nm}$ for the broad spectrum $(600 \sim 800 \mathrm{~nm})$ of $\mathrm{NV}$ centers, the value corresponds to a spatial resolution of $\sim \lambda / 2.5$, close to the Abbe diffraction limit of $\lambda /(2 \times \mathrm{NA})$, where the NA is 1.4 with an oil immersion lens. For microsphere-assisted microscopy, we measure the point-spread function at two different virtual image planes $(z=$ -13 and $-17 \mu \mathrm{m})$ and achieve fwhm values of $188 \mathrm{~nm}(\lambda / 3.7)$ and $142 \mathrm{~nm}(\lambda / 5)$, respectively. Therefore, microsphereassisted microscopy provides spatial resolution beyond the diffraction limit in conventional microscopy. Figure $3 \mathrm{~d}$ plots the spatial resolution as well as the intensity with the imaging depth from the surface. The microsphere forms the virtual image with the highest intensity at approximately $z=-12 \sim$ $-13 \mu \mathrm{m}$. As the image plane moves further down, we observe higher resolution, but lose the intensity. At $z=-17 \mu \mathrm{m}$, we achieve a resolution of approximately $\lambda / 5$, but the intensity is reduced to $20 \%$ that of the maximum intensity. Additionally, the intensity profile at $z=-17 \mu \mathrm{m}$ shows two shoulder peaks (Figure 3c). These peaks originate from airy patterns, which become stronger as we pass the focal plane $d_{\mathrm{v}}=-13 \mu \mathrm{m}$ (see Figure S4 in the Supporting Information). We note that the size of a pinhole affects both spatial resolution and signal-tonoise ratio. We selected the size of pinhole $(50 \mu \mathrm{m})$ that shows optimal resolution, signal-to-noise ratio, and brightness (see Figure S5 in the Supporting Information).

With the enhanced resolving power and improved optical signal contrast, we examine closely spaced defects, which are not resolved by conventional microscopy. In Figure 3e,f, conventional microscopy shows the intensity profile of defect group D as a single merged peak, whereas microsphere-assisted microscopy shows two separated peaks for defects D1 and D2 in Figure $3 \mathrm{~b}$. Each single quantum emitter behaves as a singlephoton source, quantified by an antibunching dip, ideally $g^{(2)}(0)=0$. When multiple defects spatially overlap, the value increases as $g^{(2)}(0)=1-\frac{1}{N}$, where $N$ is the number of quantum emitters. To investigate single-photon purity, we 

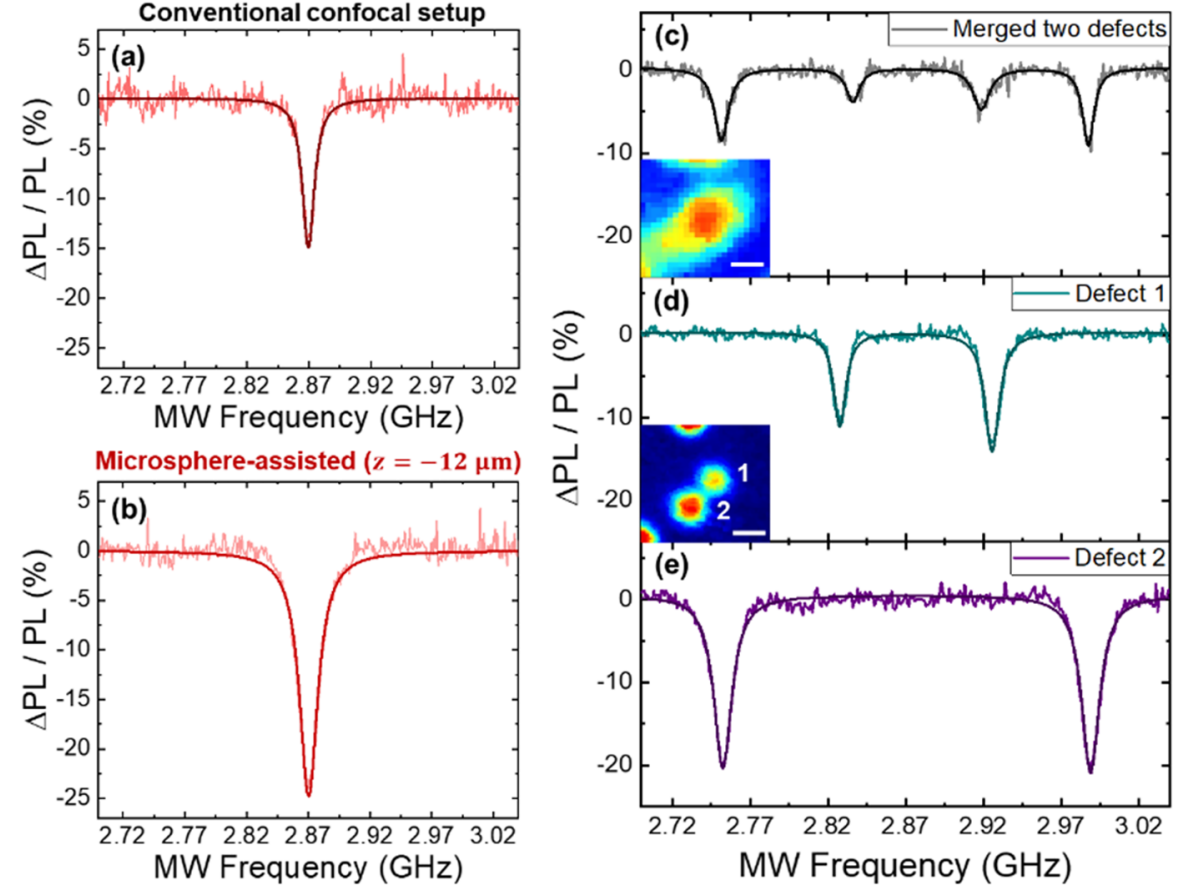

Figure 4. (a, b) ODMR spectra of the single defect (b) with and (a) without a microsphere. (c-e) ODMR spectra with a magnetic field of approximately $50 \mathrm{G}$ for (c) two spatially unresolved defects in conventional confocal microscopy and two spatially resolved defects (d) 1 and (e) 2 in microsphere-assisted microscopy. The inset images represent confocal mapping images of the same defects in conventional and microsphereassisted microscopy. Scale bars in the confocal maps denote $200 \mathrm{~nm}$.

measure the second-order photon correlation $g^{(2)}(\tau)$ using Hanbury Brown and Twiss measurements and fit the data using the following equation:

$$
g^{(2)}(\tau)=1-\left(1-g^{(2)}(0)\right)\left(C \exp \left(-\frac{|\tau|}{\tau_{1}}\right)+(1-C) \exp \left(-\frac{|\tau|}{\tau_{2}}\right)\right)
$$

where $\tau_{1(2)}$ is a time constant relevant to the lifetime of excited states (metastable state), and $C$ is a coefficient for the antibunching dip and bunching peak with a time scale of $\tau_{1(2)}$. For the single defect $(\mathrm{S})$, both conventional and microsphereassisted microscopy show expected low $g^{(2)}(0)$ values of $0.12 \pm$ 0.07 and $0.09 \pm 0.06$, respectively (see Figure S6 in the Supporting Information). Then, we measure $g^{(2)}(\tau)$ for the two closely spaced defects. For defect group $\mathrm{D}$, we record the histogram at the maximum intensity of the merged peak and achieve $g^{(2)}(0)=0.3 \pm 0.08$ (Figure $3 \mathrm{~g}$ ). The value is noticeably increased compared with that from the single defect. On the other hand, for spatially resolved single defects D1 and $\mathrm{D} 2$, the $g^{(2)}(0)$ values of each single defect D1 and D2 in the virtual image remain as low as $0.13 \pm 0.06$ and $0.16 \pm 0.06$, respectively (Figure $3 \mathrm{~h}$ ). Such high single-photon purity validates that magnified, high-resolution imaging allows optical separation of single photons from the emission of adjacent defects. It is also worth noting that the $g^{(2)}(0)$ value is still below 0.5 for two spatially overlapping defects in conventional microscopy. The emissions from two independent, identical quantum emitters are expected to have $g^{(2)}(0) \sim 0.5$. However, even with two defects, it is possible to have a $g^{(2)}(0)$ value below 0.5 when the brightness of each emitter is not identical or the measurement is spatially one-sided between two defects. In addition, these defects are temporally blinking, making them behave as a mixture of single emitter and two emitters under the given experimental conditions. The fact is that $g^{(2)}(0)<0.5$ is often used as a criterion for a single defect, but the results suggest that $g^{(2)}(0)<0.5$ alone cannot strongly support the single quantum emitters. Therefore, having a high-resolution imaging system will be much more beneficial to identify single defects and independently manipulate closely spaced defects.

Next, we demonstrate that microsphere-assisted microscopy improves the spin readout contrast and enables us to address single spins of adjacent defects independently. The NV centers in diamond have a zero-field splitting of $2.87 \mathrm{GHz}^{52}$ without an external magnetic field, which can be detected by optically detected magnetic resonance (ODMR) measurements. Figure $4 a, b$ shows the ODMR spectra of the same single defect in conventional and microsphere-assisted microscopy. For comparison, the single defect under a microsphere exhibits an ODMR contrast of $25 \pm 0.9 \%$, much higher than the value of $15 \pm 1.1 \%$ in conventional microscopy. We attribute this increase to the improved signal-to-noise ratio in the virtual image.

To prove that we can address single spins of the merged defects in a conventional optical diffraction limit, we examine the ODMR spectrum of two closely spaced defects using both microscopy setups. Although two defects spatially overlap in conventional microscopy, as shown in the inset of Figure 4c, we can confirm that two different spins exist by applying a randomly oriented magnetic field $(\sim 50 \mathrm{G})$ that breaks the degeneracy of $S=1$. Since the NV center in diamond has four possible spin configurations in the crystal, two defects can have a different amount of Zeeman splitting according to the applied magnetic field. ${ }^{53}$ In Figure 4c, we observe four peaks from two defects in the ODMR spectrum. Each defect shows splittings of $\delta_{1}= \pm 50 \mathrm{MHz}$ and $\delta_{2}= \pm 118 \mathrm{MHz}$ from the center at $2.87 \mathrm{GHz}$. Since these defects are merged in conventional microscopy, we cannot optically address each spin. However, with microsphere-assisted confocal microscopy, these defects are clearly resolved, as shown in the inset of 
Figure 4d. We record the ODMR signals from each defect, as shown in Figure 4d,e. In contrast to the previous four peaks from the two unresolved defects, each ODMR spectrum shows only two separated peaks from each defect $\left(\delta_{1}= \pm 55 \mathrm{MHz}\right.$ for defect 1 and $\delta_{2}= \pm 120 \mathrm{MHz}$ for defect 2) without crosstalk from another defect. The small difference in $\delta_{1,2}$ between the two setups is due to the slight change in magnet alignment during the measurements. Therefore, demonstrating enhanced spatial resolution and signal contrast, this microsphere-assisted imaging approach allows us to independently address single photons and single spins of adjacent defects that are spatially unresolved in conventional microscopy.

\section{CONCLUSIONS}

In conclusion, we have demonstrated a high-resolution and high-contrast optical interface that independently addresses single photons and single spins of defects that appear merged in conventional microscopy. The microspheres showed great potential as an efficient optical interface for defects, with a subdiffraction-limited spatial resolution up to $\sim \lambda / 5$ and a signal-to-noise ratio improvement of more than four times compared to that of conventional confocal microscopy. The spatial resolution of microsphere-assisted microscopy can be improved further. A $100 \mu \mathrm{m}$ sized microsphere has shown a resolution of $\sim \lambda / 7$ that resolves $75 \mathrm{~nm}$ sized features, ${ }^{37}$ and combining plasmonic structures can strengthen the evanescent field, increasing the resolution up to $\sim \lambda / 10 .^{51}$ The size of the photonic nanoject can also be narrowed down further by resonantly driving whispering gallery modes of the microsphere $^{43}$ or coupling multiple microspheres. ${ }^{54}$ Besides, the chromatic aberration in microspheres also plays a role in spatial resolution. These chromatic aberrations could be reduced by employing dielectric microspheres with low spectral dispersion and imaging the defects having narrow line width, such as $\mathrm{SiV}$ in diamond.

The microsphere can be easily adapted to conventional optical setups, and compatible with various environments (oil, water, and air) and objects (nanoparticles, biomolecule, and thin films). Therefore, the technique can simply enhance the resolution and sensitivity of quantum sensing and imaging applications with defects. ${ }^{9,12,13,55,56}$ Additionally, the capability of addressing adjacent defects independently is essential to manipulate multiple defects and their interactions. For example, when identical emitters are closely spaced less than their wavelength scale, they can create an entangled superposition state, showing the collective behavior of superradiance. ${ }^{57-59}$ Since conventional microscopy is limited to addressing individual emitters spaced in such a short distance, the previous works mostly introduced single-mode waveguides or cavities to extend the interaction range. ${ }^{7,60,61}$ Our result of selective excitation and control of individual defects within a subwavelength scale shows the possibility of deterministic addressing of these interactions.

The integration of microspheres does not use any fabrication process, but it can be simply adapted with various defects in bulk crystals and conventional microscopy systems. Furthermore, the possibility of relocating these micro-optics extends their uses for scanning and deterministic positioning for defects. Therefore, microsphere-assisted microscopy will pave the way for exploring solid-state quantum systems.

\section{ASSOCIATED CONTENT}

\section{Supporting Information}

The Supporting Information is available free of charge at https://pubs.acs.org/doi/10.1021/acsphotonics.1c00576.

Comparison of the intensity of a single defect with/ without a microsphere; simulation of position-dependent intensity enhancement; Depth-dependent virtual images; Comparison of the second-order correlation of single defects; Optical positioning of microspheres (PDF)

Movie of optical positioning of microspheres (AVI)

\section{AUTHOR INFORMATION}

\section{Corresponding Author}

Je-Hyung Kim - School of Nature Science, Department of Physics, Ulsan National Institute of Science and Technology (UNIST), Ulsan 44919, Republic of Korea; 이이.org/ 0000-0002-6894-9285; Email: jehyungkim@unist.ac.kr

\section{Authors}

Jong Sung Moon - School of Nature Science, Department of Physics, Ulsan National Institute of Science and Technology (UNIST), Ulsan 44919, Republic of Korea

Haneul Lee - School of Nature Science, Department of Physics, Ulsan National Institute of Science and Technology (UNIST), Ulsan 44919, Republic of Korea

Jin Hee Lee - School of Nature Science, Department of Physics, Ulsan National Institute of Science and Technology (UNIST), Ulsan 44919, Republic of Korea

Woong Bae Jeon - School of Nature Science, Department of Physics, Ulsan National Institute of Science and Technology (UNIST), Ulsan 44919, Republic of Korea

Dowon Lee - School of Nature Science, Department of Physics, Ulsan National Institute of Science and Technology (UNIST), Ulsan 44919, Republic of Korea

Junghyun Lee - Center for Quantum Information, Korea Institute of Science and Technology, Seoul 02792, Republic of Korea

Seoyoung Paik - Center for Quantum Information, Korea Institute of Science and Technology, Seoul 02792, Republic of Korea; Department of Physics and Photon Science, Gwangju Institute of Science and Technology, Gwangju 61005, Republic of Korea

Sang-Wook Han - Center for Quantum Information, Korea Institute of Science and Technology, Seoul 02792, Republic of Korea

Rolf Reuter - 3. Physikalisches Institut and Research Center SCOPE and Integrated Quantum Science and Technology (IQST), University of Stuttgart, 70569 Stuttgart, Germany

Andrej Denisenko - 3. Physikalisches Institut and Research Center SCOPE and Integrated Quantum Science and Technology (IQST), University of Stuttgart, 70569 Stuttgart, Germany

Jörg Wrachtrup - 3. Physikalisches Institut and Research Center SCOPE and Integrated Quantum Science and Technology (IQST), University of Stuttgart, 70569 Stuttgart, Germany

Sang-Yun Lee - Center for Quantum Information, Korea Institute of Science and Technology, Seoul 02792, Republic of Korea; Department of Physics and Photon Science, Gwangju Institute of Science and Technology, Gwangju 61005, Republic of Korea; ○ orcid.org/0000-0003-1777-980X 
Complete contact information is available at:

https://pubs.acs.org/10.1021/acsphotonics.1c00576

\section{Author Contributions}

${ }^{\ddagger}$ J.S.M. and H.L. contributed equally to this work.

\section{Notes}

The authors declare no competing financial interest.

\section{ACKNOWLEDGMENTS}

This work is supported by the National Research Foundation of Korea (MSIT; NRF-2019M3E4A1078664, NRF2020M3H3A1098869); Institute of Information and Communications Technology Planning and Evaluation (IITP) Grant (2019-0-00434); the ITRC (Information Technology Research Center) support program (IITP-2021-2020-0-01606) supervised by the IITP; the KIST Institutional Program (2E3102121-032); and the GIST Research Institute(GRI) grant funded by the GIST in 2020 .

\section{REFERENCES}

(1) Atatüre, M.; Englund, D.; Vamivakas, N.; Lee, S.-Y.; Wrachtrup, J. Material platforms for spin-based photonic quantum technologies. Nat. Rev. Mater. 2018, 3 (5), 38-51.

(2) Pezzagna, S.; Meijer, J. Quantum computer based on color centers in diamond. Appl. Phys. Rev. 2021, 8 (1), No. 011308.

(3) Balasubramanian, G.; Neumann, P.; Twitchen, D.; Markham, M.; Kolesov, R.; Mizuochi, N.; Isoya, J.; Achard, J.; Beck, J.; Tissler, J.; Jacques, V.; Hemmer, P. R.; Jelezko, F.; Wrachtrup, J. Ultralong spin coherence time in isotopically engineered diamond. Nat. Mater. 2009, 8 (5), 383-387.

(4) Ishikawa, T.; Fu, K.-M. C.; Santori, C.; Acosta, V. M.; Beausoleil, R. G.; Watanabe, H.; Shikata, S.; Itoh, K. M. Optical and Spin Coherence Properties of Nitrogen-Vacancy Centers Placed in a 100 $\mathrm{nm}$ Thick Isotopically Purified Diamond Layer. Nano Lett. 2012, 12 (4), 2083-2087.

(5) Neumann, P.; Kolesov, R.; Naydenov, B.; Beck, J.; Rempp, F.; Steiner, M.; Jacques, V.; Balasubramanian, G.; Markham, M. L.; Twitchen, D. J.; Pezzagna, S.; Meijer, J.; Twamley, J.; Jelezko, F.; Wrachtrup, J. Quantum register based on coupled electron spins in a room-temperature solid. Nat. Phys. 2010, 6 (4), 249-253.

(6) Bradley, C. E.; Randall, J.; Abobeih, M. H.; Berrevoets, R. C.; Degen, M. J.; Bakker, M. A.; Markham, M.; Twitchen, D. J.; Taminiau, T. H. A Ten-Qubit Solid-State Spin Register with Quantum Memory up to One Minute. Phys. Rev. X 2019, 9 (3), No. 031045 .

(7) Angerer, A.; Streltsov, K.; Astner, T.; Putz, S.; Sumiya, H.; Onoda, S.; Isoya, J.; Munro, W. J.; Nemoto, K.; Schmiedmayer, J.; Majer, J. Superradiant emission from colour centres in diamond. Nat. Phys. 2018, 14 (12), 1168-1172.

(8) Bradac, C.; Johnsson, M. T.; Breugel, M. v.; Baragiola, B. Q.; Martin, R.; Juan, M. L.; Brennen, G. K.; Volz, T. Room-temperature spontaneous superradiance from single diamond nanocrystals. Nat. Commun. 2017, 8 (1), 1205.

(9) Foy, C.; Zhang, L.; Trusheim, M. E.; Bagnall, K. R.; Walsh, M.; Wang, E. N.; Englund, D. R. Wide-Field Magnetic Field and Temperature Imaging Using Nanoscale Quantum Sensors. ACS Appl. Mater. Interfaces 2020, 12 (23), 26525-26533.

(10) Mizuno, K.; Ishiwata, H.; Masuyama, Y.; Iwasaki, T.; Hatano, M. Simultaneous wide-field imaging of phase and magnitude of AC magnetic signal using diamond quantum magnetometry. Sci. Rep. 2020, $10(1), 11611$.

(11) Dolde, F.; Fedder, H.; Doherty, M. W.; Nöbauer, T.; Rempp, F.; Balasubramanian, G.; Wolf, T.; Reinhard, F.; Hollenberg, L. C. L.; Jelezko, F.; Wrachtrup, J. Electric-field sensing using single diamond spins. Nat. Phys. 2011, 7 (6), 459-463.
(12) Tetienne, J.-P.; Dontschuk, N.; Broadway, D. A.; Stacey, A.; Simpson, D. A.; Hollenberg, L. C. L. Quantum imaging of current flow in graphene. Sci. Adv. 2017, 3 (4), No. e1602429.

(13) Le Sage, D.; Arai, K.; Glenn, D. R.; DeVience, S. J.; Pham, L. M.; Rahn-Lee, L.; Lukin, M. D.; Yacoby, A.; Komeili, A.; Walsworth, R. L. Optical magnetic imaging of living cells. Nature 2013, 496 (7446), 486-489.

(14) Babinec, T. M.; Hausmann, B. J. M.; Khan, M.; Zhang, Y.; Maze, J. R.; Hemmer, P. R.; Lončar, M. A diamond nanowire singlephoton source. Nat. Nanotechnol. 2010, 5 (3), 195-199.

(15) Hausmann, B. J. M.; Shields, B. J.; Quan, Q.; Chu, Y.; de Leon, N. P.; Evans, R.; Burek, M. J.; Zibrov, A. S.; Markham, M.; Twitchen, D. J.; Park, H.; Lukin, M. D.; Loncăr, M. Coupling of NV Centers to Photonic Crystal Nanobeams in Diamond. Nano Lett. 2013, 13 (12), 5791-5796.

(16) Li, L.; Chen, E. H.; Zheng, J.; Mouradian, S. L.; Dolde, F.; Schröder, T.; Karaveli, S.; Markham, M. L.; Twitchen, D. J.; Englund, D. Efficient Photon Collection from a Nitrogen Vacancy Center in a Circular Bullseye Grating. Nano Lett. 2015, 15 (3), 1493-1497.

(17) Chakravarthi, S.; Chao, P.; Pederson, C.; Molesky, S.; Ivanov, A.; Hestroffer, K.; Hatami, F.; Rodriguez, A. W.; Fu, K.-M. C. Inversedesigned photon extractors for optically addressable defect qubits. Optica 2020, 7 (12), 1805-1811.

(18) Barnes, W. L.; Björk, G.; Gérard, J. M.; Jonsson, P.; Wasey, J. A. E.; Worthing, P. T.; Zwiller, V. Solid-state single photon sources: light collection strategies. Eur. Phys. J. D 2002, 18 (2), 197-210.

(19) Bradac, C.; Gaebel, T.; Naidoo, N.; Sellars, M. J.; Twamley, J.; Brown, L. J.; Barnard, A. S.; Plakhotnik, T.; Zvyagin, A. V.; Rabeau, J. R. Observation and control of blinking nitrogen-vacancy centres in discrete nanodiamonds. Nat. Nanotechnol. 2010, 5 (5), 345-349.

(20) Faraon, A.; Santori, C.; Huang, Z.; Acosta, V. M.; Beausoleil, R. G. Coupling of Nitrogen-Vacancy Centers to Photonic Crystal Cavities in Monocrystalline Diamond. Phys. Rev. Lett. 2012, 109 (3), No. 033604.

(21) Tisler, J.; Balasubramanian, G.; Naydenov, B.; Kolesov, R.; Grotz, B.; Reuter, R.; Boudou, J.-P.; Curmi, P. A.; Sennour, M.; Thorel, A.; Börsch, M.; Aulenbacher, K.; Erdmann, R.; Hemmer, P. R.; Jelezko, F.; Wrachtrup, J. Fluorescence and Spin Properties of Defects in Single Digit Nanodiamonds. ACS Nano 2009, 3 (7), 1959-1965.

(22) Albrecht, R.; Bommer, A.; Deutsch, C.; Reichel, J.; Becher, C. Coupling of a Single Nitrogen-Vacancy Center in Diamond to a Fiber-Based Microcavity. Phys. Rev. Lett. 2013, 110 (24), 243602.

(23) Riedel, D.; Söllner, I.; Shields, B. J.; Starosielec, S.; Appel, P.; Neu, E.; Maletinsky, P.; Warburton, R. J. Deterministic Enhancement of Coherent Photon Generation from a Nitrogen-Vacancy Center in Ultrapure Diamond. Phys. Rev. X 2017, 7 (3), No. 031040.

(24) van der Sar, T.; Heeres, E. C.; Dmochowski, G. M.; de Lange, G.; Robledo, L.; Oosterkamp, T. H.; Hanson, R. Nanopositioning of a diamond nanocrystal containing a single nitrogen-vacancy defect center. Appl. Phys. Lett. 2009, 94 (17), 173104.

(25) Fukuda, R.; Balasubramanian, P.; Higashimata, I.; Koike, G.; Okada, T.; Kagami, R.; Teraji, T.; Onoda, S.; Haruyama, M.; Yamada, K.; Inaba, M.; Yamano, H.; Stürner, F. M.; Schmitt, S.; McGuinness, L. P.; Jelezko, F.; Ohshima, T.; Shinada, T.; Kawarada, H.; Kada, W.; Hanaizumi, O.; Tanii, T.; Isoya, J. Lithographically engineered shallow nitrogen-vacancy centers in diamond for external nuclear spin sensing. New J. Phys. 2018, 20 (8), No. 083029.

(26) Betzig, E.; Chichester, R. J. Single Molecules Observed by Near-Field Scanning Optical Microscopy. Science 1993, 262 (5138), $1422-1425$.

(27) Willets, K. A.; Wilson, A. J.; Sundaresan, V.; Joshi, P. B. SuperResolution Imaging and Plasmonics. Chem. Rev. 2017, 117 (11), $7538-7582$.

(28) Pezzagna, S.; Wildanger, D.; Mazarov, P.; Wieck, A. D.; Sarov, Y.; Rangelow, I.; Naydenov, B.; Jelezko, F.; Hell, S. W.; Meijer, J. Nanoscale Engineering and Optical Addressing of Single Spins in Diamond. Small 2010, 6 (19), 2117-2121. 
(29) Rittweger, E.; Han, K. Y.; Irvine, S. E.; Eggeling, C.; Hell, S. W. STED microscopy reveals crystal colour centres with nanometric resolution. Nat. Photonics 2009, 3 (3), 144-147.

(30) Arai, K.; Belthangady, C.; Zhang, H.; Bar-Gill, N.; DeVience, S. J.; Cappellaro, P.; Yacoby, A.; Walsworth, R. L. Fourier magnetic imaging with nanoscale resolution and compressed sensing speed-up using electronic spins in diamond. Nat. Nanotechnol. 2015, 10 (10), 859-864.

(31) Wan, N. H.; Shields, B. J.; Kim, D.; Mouradian, S.; Lienhard, B.; Walsh, M.; Bakhru, H.; Schröder, T.; Englund, D. Efficient Extraction of Light from a Nitrogen-Vacancy Center in a Diamond Parabolic Reflector. Nano Lett. 2018, 18 (5), 2787-2793.

(32) Hadden, J. P.; Harrison, J. P.; Stanley-Clarke, A. C.; Marseglia, L.; Ho, Y. L. D.; Patton, B. R.; O’Brien, J. L.; Rarity, J. G. Strongly enhanced photon collection from diamond defect centers under microfabricated integrated solid immersion lenses. Appl. Phys. Lett. 2010, 97 (24), 241901.

(33) Siyushev, P.; Kaiser, F.; Jacques, V.; Gerhardt, I.; Bischof, S.; Fedder, H.; Dodson, J.; Markham, M.; Twitchen, D.; Jelezko, F.; Wrachtrup, J. Monolithic diamond optics for single photon detection. Appl. Phys. Lett. 2010, 97 (24), 241902.

(34) Robledo, L.; Childress, L.; Bernien, H.; Hensen, B.; Alkemade, P. F. A.; Hanson, R. High-fidelity projective read-out of a solid-state spin quantum register. Nature 2011, 477 (7366), 574-578.

(35) Wu, Q.; Feke, G. D.; Grober, R. D.; Ghislain, L. P. Realization of numerical aperture 2.0 using a gallium phosphide solid immersion lens. Appl. Phys. Lett. 1999, 75 (26), 4064-4066.

(36) Jamali, M.; Gerhardt, I.; Rezai, M.; Frenner, K.; Fedder, H.; Wrachtrup, J. Microscopic diamond solid-immersion-lenses fabricated around single defect centers by focused ion beam milling. Rev. Sci. Instrum. 2014, 85 (12), 123703.

(37) Li, L.; Guo, W.; Yan, Y.; Lee, S.; Wang, T. Label-free superresolution imaging of adenoviruses by submerged microsphere optical nanoscopy. Light: Sci. Appl. 2013, 2 (9), e104-e104.

(38) Zhu, H.; Fan, W.; Zhou, S.; Chen, M.; Wu, L. Polymer Colloidal Sphere-Based Hybrid Solid Immersion Lens for Optical Super-resolution Imaging. ACS Nano 2016, 10 (10), 9755-9761.

(39) Wang, Z.; Guo, W.; Li, L.; Luk'yanchuk, B.; Khan, A.; Liu, Z.; Chen, Z.; Hong, M. Optical virtual imaging at $50 \mathrm{~nm}$ lateral resolution with a white-light nanoscope. Nat. Commun. 2011, 2 (1), 218.

(40) Wang, Z.; Luk'yanchuk, B. Super-resolution imaging and microscopy by dielectric particle-lenses. In Label-Free Super-Resolution Microscopy; Springer, 2019; pp 371-406.

(41) Lai, H. S. S.; Wang, F.; Li, Y.; Jia, B.; Liu, L.; Li, W. J. Superresolution real imaging in microsphere-assisted microscopy. PLoS One 2016, 11 (10), No. e0165194.

(42) Wang, F.; Yang, S.; Ma, H.; Shen, P.; Wei, N.; Wang, M.; Xia, Y.; Deng, Y.; Ye, Y.-H. Microsphere-assisted super-resolution imaging with enlarged numerical aperture by semi-immersion. Appl. Phys. Lett. 2018, 112 (2), No. 023101.

(43) Maslov, A. V.; Astratov, V. N. Resolution and Reciprocity in Microspherical Nanoscopy: Point-Spread Function Versus Photonic Nanojets. Phys. Rev. Appl. 2019, 11 (6), No. 064004.

(44) Yang, H.; Trouillon, R.; Huszka, G.; Gijs, M. A. M. SuperResolution Imaging of a Dielectric Microsphere Is Governed by the Waist of Its Photonic Nanojet. Nano Lett. 2016, 16 (8), 4862-4870.

(45) Darafsheh, A.; Walsh, G. F.; Dal Negro, L.; Astratov, V. N. Optical super-resolution by high-index liquid-immersed microspheres. Appl. Phys. Lett. 2012, 101 (14), 141128.

(46) Chen, Z.; Taflove, A.; Backman, V. Photonic nanojet enhancement of backscattering of light by nanoparticles: a potential novel visible-light ultramicroscopy technique. Opt. Express 2004, 12 (7), 1214-1220.

(47) Zhu, J.; Goddard, L. L. All-dielectric concentration of electromagnetic fields at the nanoscale: the role of photonic nanojets. Nanoscale Adv. 2019, 1 (12), 4615-4643.

(48) Wang, F.; Liu, L.; Yu, H.; Wen, Y.; Yu, P.; Liu, Z.; Wang, Y.; Li, W. J. Scanning superlens microscopy for non-invasive large field-of- view visible light nanoscale imaging. Nat. Commun. 2016, 7 (1), 13748 .

(49) Krivitsky, L. A.; Wang, J. J.; Wang, Z.; Luk'yanchuk, B. Locomotion of microspheres for super-resolution imaging. Sci. Rep. 2013, 3 (1), 3501.

(50) McLeod, E.; Arnold, C. B. Subwavelength direct-write nanopatterning using optically trapped microspheres. Nat. Nanotechnol. 2008, 3 (7), 413-417.

(51) Bezryadina, A.; Li, J.; Zhao, J.; Kothambawala, A.; Ponsetto, J.; Huang, E.; Wang, J.; Liu, Z. Localized plasmonic structured illumination microscopy with an optically trapped microlens. Nanoscale 2017, 9 (39), 14907-14912.

(52) Gruber, A.; Dräbenstedt, A.; Tietz, C.; Fleury, L.; Wrachtrup, J.; Borczyskowski, C. v. Scanning Confocal Optical Microscopy and Magnetic Resonance on Single Defect Centers. Science 1997, 276 (5321), 2012.

(53) Pham, L. M.; Bar-Gill, N.; Le Sage, D.; Belthangady, C.; Stacey, A.; Markham, M.; Twitchen, D. J.; Lukin, M. D.; Walsworth, R. L. Enhanced metrology using preferential orientation of nitrogenvacancy centers in diamond. Phys. Rev. B: Condens. Matter Mater. Phys. 2012, 86 (12), 121202.

(54) Devilez, A.; Wenger, J.; Stout, B.; Bonod, N. Transverse and longitudinal confinement of photonic nanojets by compound dielectric microspheres. Proc. SPIE 2009, 73930E.

(55) Casola, F.; van der Sar, T.; Yacoby, A. Probing condensed matter physics with magnetometry based on nitrogen-vacancy centres in diamond. Nat. Rev. Mater. 2018, 3 (1), 17088.

(56) Schmitt, S.; Gefen, T.; Stürner, F. M.; Unden, T.; Wolff, G.; Müller, C.; Scheuer, J.; Naydenov, B.; Markham, M.; Pezzagna, S.; Meijer, J.; Schwarz, I.; Plenio, M.; Retzker, A.; McGuinness, L. P.; Jelezko, F. Submillihertz magnetic spectroscopy performed with a nanoscale quantum sensor. Science 2017, 356 (6340), 832-837.

(57) Scheibner, M.; Schmidt, T.; Worschech, L.; Forchel, A.; Bacher, G.; Passow, T.; Hommel, D. Superradiance of quantum dots. Nat. Phys. 2007, 3 (2), 106-110.

(58) Dicke, R.; Coherence, H. in Spontaneous Radiation Processes. Phys. Rev. 1954, 93 (1), 99-110.

(59) Gross, M.; Haroche, S. Superradiance: An essay on the theory of collective spontaneous emission. Phys. Rep. 1982, 93 (5), 301-396.

(60) Kim, J.-H.; Aghaeimeibodi, S.; Richardson, C. J. K.; Leavitt, R. P.; Waks, E. Super-Radiant Emission from Quantum Dots in a Nanophotonic Waveguide. Nano Lett. 2018, 18 (8), 4734-4740.

(61) Evans, R. E.; Bhaskar, M. K.; Sukachev, D. D.; Nguyen, C. T.; Sipahigil, A.; Burek, M. J.; Machielse, B.; Zhang, G. H.; Zibrov, A. S.; Bielejec, E.; Park, H.; Lončar, M.; Lukin, M. D. Photon-mediated interactions between quantum emitters in a diamond nanocavity. Science 2018, 362 (6415), 662. 